\title{
Sensibilité aux Insecticides des Populations de Bemisia tabaci (Gennadius, 1889) Collectées dans Différentes Zones de Production Cotonnière en Côte d'Ivoire
}

\section{Gouzou Juste Roland Didi,}

University Nangui Abrogoua, Laboratory of Animal Cytology and Biology,

Abidjan, Côte d'Ivoire

National Center of Agronomic Research, Cotton Research Station, Laboratory of Entomology, Bouaké, Côte d'Ivoire

\section{Malanno Kouakou,}

National Center of Agronomic Research, Cotton Research Station,

Laboratory of Entomology,

Pitou Woklin Euloge Koné,

Germain Elisabeth Cynthia Ochou,

University Nangui Abrogoua, Laboratory of Animal Cytology and Biology,

Abidjan, Côte d'Ivoire

National Center of Agronomic Research, Cotton Research Station,

Laboratory of Entomology, Bouaké, Côte d'Ivoire

\section{Kouadio Kra Norbert Bini, Ochou Germain Ochou,}

National Center of Agronomic Research, Cotton Research Station, Laboratory of Entomology, Bouaké, Côte d'Ivoire

\section{Dagnogo Mamadou,}

University Nangui Abrogoua, Laboratory of Animal Cytology and Biology, Abidjan, Côte d'Ivoire

\section{Résumé}

Ces dernières années, il a été constaté en Côte d'Ivoire une pullulation des populations de Bemisia tabaci en culture cotonnière malgré les traitements insecticides effectués. Une évaluation des niveaux de sensibilité aux insecticides couramment utilisés pour la protection des cotonniers a été entreprise en vue d'identifier les matières actives les plus efficaces contre $B$. tabaci. Des populations de mouches blanches ont été collectées dans six (6) différentes localités de la zone de production cotonnière (Niakara, Bouaké, Ouangolo, Ferké, Boundiali et Korhogo). Elles ont été testées par la méthode de trempage des rondelles de feuilles vis-à-vis de cinq (5) insecticides de 
familles chimiques différentes (Organophosphorés, Pyréthrinoïdes, Néonicotinoïdes, Sulfoximines, Spinosynes). Les $\mathrm{CL}_{50}$ pour chaque insecticide ont été calculées grâce au modèle probit-log et comparées à l'aide d'analyses univariées de variance (ANOVA). Les données ont montré une variabilité géographique de la sensibilité aux insecticides des populations de $B$. tabaci et une différence significative entre les niveaux de sensibilité de l'insecte vis-à-vis des insecticides testés. Les souches provenant de Korhogo ont présenté la plus faible sensibilité au profenofos $\left(\mathrm{CL}_{50}=71,51 \mathrm{mg} . \mathrm{l}^{-1}\right)$, et celles de Boundiali la plus faible sensibilité à l'acétamipride $\left(\mathrm{CL}_{50}=219,96\right.$ $\left.\mathrm{mg} . \mathrm{l}^{-1}\right)$.L'acétamipride $\mathrm{s}$ 'est montré significativement moins toxique comparativement à la lambdacyhalothrine et au profenofos $(\mathrm{F}=2,58$; $\mathrm{P}=0,04)$. Le sulfoxaflor $\left(\mathrm{CL}_{50}\right.$ moyenne $\left.=0,71 \mathrm{mg} . \mathrm{l}^{-1}\right)$ et le spinosad $\left(\mathrm{CL}_{50}\right.$ moyenne $=4,02 \mathrm{mg} .1^{-1}$ ) ont été les produits les plus toxiques. Cette étude qui est la première du genre en Côte d'Ivoire représente une base de données pour la gestion des populations de $B$. tabaci.

Mots clés : Côte d'Ivoire, Bemisia Tabaci, Sensibilité, Insecticides, Coton 


\section{Insecticide Susceptibility of Bemisia Tabaci (Gennadius, 1889) Populations Collected from Different Cotton-Producing Areas in Côte d'Ivoire}

\section{Gouzou Juste Roland Didi,}

University Nangui Abrogoua, Laboratory of Animal Cytology and Biology,

Abidjan, Côte d'Ivoire

National Center of Agronomic Research, Cotton Research Station, Laboratory of Entomology, Bouaké, Côte d'Ivoire

\section{Malanno Kouakou,}

National Center of Agronomic Research, Cotton Research Station,

Laboratory of Entomology,

Pitou Woklin Euloge Koné,

Germain Elisabeth Cynthia Ochou,

University Nangui Abrogoua, Laboratory of Animal Cytology and Biology,

Abidjan, Côte d'Ivoire

National Center of Agronomic Research, Cotton Research Station,

Laboratory of Entomology, Bouaké, Côte d'Ivoire

\section{Kouadio Kra Norbert Bini, Ochou Germain Ochou,}

National Center of Agronomic Research, Cotton Research Station, Laboratory of Entomology, Bouaké, Côte d'Ivoire

Dagnogo Mamadou,

University Nangui Abrogoua, Laboratory of Animal Cytology and Biology, Abidjan, Côte d'Ivoire

\section{Abstract}

In recent years, an outbreak of Bemisia tabaci has been observed in Côte d'Ivoire in cotton crops despite the insecticide treatments. An evaluation of the sensitivity levels of insecticides commonly used for the protection of cotton trees has been undertaken in order to identify the most effective active ingredients against $B$. tabaci. Whitefly populations were collected in six (6) different localities in the cotton production area (Niakara, Bouaké, Ouangolo, Ferké, Boundiali and Korhogo). They were tested by the leaf dipping method with five (5) insecticides of different chemical families (Organophosphorus, Pyrethroids, Neonicotinoids, Sulfoximines, Spinosynes). LC $_{50}$ s for each insecticide were calculated using the probit-log model and compared using 
univariate analyses of variance (ANOVA). The data showed a geographical variability in insecticide susceptibility of $B$. tabaci populations and a significant difference between the levels of insect susceptibility to the insecticides tested. Strains from Korhogo showed the lowest susceptibility to profenofos $\left(\mathrm{LC}_{50}=71.51 \mathrm{mg} . \mathrm{l}^{-1}\right)$, and strains from Boundiali the lowest susceptibility to acetamiprid $\left(\mathrm{LC}_{50}=219.96 \mathrm{mg}^{-\mathrm{l}^{-1}}\right)$. Acétamipride was significantly less toxic compared to lambdacyhalothrin and profenofos $(\mathrm{F}=2.58 ; \mathrm{P}=0.04)$. Sulfoxaflor $\left(\mathrm{LC}_{50}=0.71 \mathrm{mg} . \mathrm{l}^{-1}\right)$ and spinosad $\left(\mathrm{LC}_{50}=4.02\right.$ mg. $1^{-1}$ ) were the most toxic products. This study, which is the first of its kind in Côte d'Ivoire, represents a database for the management of $B$. tabaci populations.

Keywords: Côte d'Ivoire, Bemisia Tabaci, Susceptibility, Insecticides, Cotton

\section{Introduction}

L'aleurode Bemisia tabaci (Gennadius, 1889) est un insecte ravageur polyphage au statut de peste mondial. L'insecte a une forte valence écologique qui lui permet de coloniser n'importe quel agrosystème (Quintela et al., 2016). Dans les champs de coton, $B$. tabaci est redouté par les dégâts impressionnants causés. Ceux-ci se caractérisent par un affaiblissement des plants attaqués, la transmission de maladies virales et la dépréciation de la qualité de la fibre de coton par le phénomène de « coton collant » dû à la présence de fumagine. Ces dégâts ont été estimés à plusieurs millions de dollar dans plusieurs pays à travers les continents asiatique, américain, européen et africain (Oliveira, Henneberry, \& Anderson, P., 2001). En Afrique de l'Ouest, les dégâts causés à la culture cotonnière par $B$. tabaci ont commencé à avoir une importance économiquement significative depuis la fin des années 90 au Mali, au Burkina Faso et en Côte d'Ivoire ( Otoidobiga, Vincent, \& Stewart, 2003). Particulièrement en Côte d'Ivoire, les infestations de l'aleurode ont eu un caractère spectaculaire au cours des campagnes cotonnières 2014 et 2015 (Didi et al., 2018). Bien que cela n'ait encore affecté la production nationale, B. tabaci reste tout de même une menace et inquiète l'ensemble des acteurs de la filière cotonnière. Cette invasion biologique de B. tabaci peut trouver son explication dans l'utilisation intensive des insecticides conventionnels (à large spectre d'action) durant plusieurs années dans les programmes de protection phytosanitaire (Horowitz et al., 2007). Aussi, cette situation de prolifération peut être imputable à une réduction du niveau des populations d'ennemis naturels de B. tabaci (Eveleens, 1983; Gnankiné et al., 2007) ou à une acquisition de la résistance aux insecticides chez les populations de l'insecte. En ce qui concerne le phénomène de résistance chez $B$. tabaci, la littérature indique plusieurs cas à travers plusieurs pays sur tous les continents. Une résistance aux pyréthrinoïdes et aux organophosphorés a été observé chez des 
populations provenant d'Espagne, des Etats unis, du Pakistan et d'Israël (Cahill et al., 1995). Il a été également observé une résistance aux néonicotinoïdes des populations de B. tabaci dans le Sud des Etat unis d'Amérique (Prabhaker et al., 2005). En Afrique du Nord, une résistance des populations égyptiennes aux carbamates, pyréthrinoïdes et organophosphorés a été mise en évidence (Kady \& Devine. 2003 ; Farghly, 2013). Des études similaires en Afrique de l'Ouest (Burkina Fasso, Togo et Benin) ont révélé une résistance des populations de $B$. tabaci à ces mêmes familles de produits chimiques couramment utilisés (Gnankiné et $a l$., 2002 ; Otoidobiga et $a l$, 2003a ; Houndété et al., 2010 ; Gnankiné et al., 2013). En Côte d'Ivoire, les études de sensibilité aux insecticides chez $B$. tabaci sont quasi inexistantes. Néanmoins, l'une des rares études de menée a porté sur les extraits de plantes à propriétés insecticides (Tia et al., 2013).

La présente étude intervient donc dans un contexte de modification du cortège parasitaire du cotonnier où $B$. tabaci se trouve être un ravageur émergent (Vaissayre et al., 2006 ; Didi et $a l ., 2018$ ) dont les niveaux d'infestation se sont accrus ces dernières années en Côte d'Ivoire, malgré les traitements chimiques effectués. Il s'agit de déterminer chez B. tabaci les niveaux de sensibilité aux insecticides couramment utilisés en vue d'identifier les produits insecticides efficaces. Ce qui permettrait d'améliorer les programmes de protection phytosanitaire qui présentent quelques insuffisances face à la prolifération de B. tabaci.

\section{Matériel et méthodes}

\section{Collecte de mouches blanches $B$. tabaci}

Les échantillons de mouches blanches ont été collectés en 2016 et 2017 dans six (6) localités de la zone de production cotonnière dont deux (2) dans la région cotonnière sud (Niakara $\left(8^{\circ} 32^{\prime} 18.773^{\prime \prime}\right.$ nord, $5^{\circ} 13^{\prime} 52.129^{\prime \prime}$ ouest) et

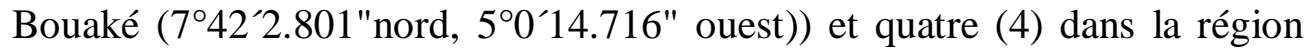
cotonnière nord (Ouangolo (10³’20.308"nord, 5²1'17.028" ouest), Ferké


$6^{\circ} 25^{\prime} 21.885^{\prime \prime}$ ouest) et Korhogo (9³0’57.931"nord, 5³6’38.21" ouest)) (figure 1). La collecte des mouches blanches s'est déroulée aussi bien en milieu paysan que sur des postes d'observations qui sont des sites expérimentaux. Les insectes ont été prélevés après l'arrêt des traitements insecticides à l'ouverture des capsules de coton. Celle-ci a lieu d'octobre à novembre au Nord et de novembre à décembre au Sud, du fait d'un décalage de saison suivant le transect Nord-Sud. Les insectes sont prélevés à l'aide d'aspirateurs à bouche et sont introduits dans des cages de dimension $70 \mathrm{~cm} \mathrm{x}$ $40 \mathrm{~cm}$ x $40 \mathrm{~cm}$. Chaque cage contient un pot avec 2 plants de coton âgés de 30 à 45 jours. Ces plants de coton n'ont subi aucun traitement insecticide. Les 
insectes ont été ensuite transportés en laboratoire et utilisés pour les tests toxicologiques 2 à $3 \mathrm{~h}$ après transport.

\section{Insecticides utilisés pour les tests toxicologiques}

Le tableau 1 indique tous les insecticides utilisés pour la réalisation des tests toxicologiques. Les matières actives mentionnées sont dans des formulations commerciales qui interviennent pour la plupart dans le programme de protection phytosanitaire en vigueur en Côte d'Ivoire. Ce programme de protection phytosanitaire est basé sur la gestion de la résistance de Helicoverpa armigera aux pyréthrinoïdes (PGRI). Il préconise six (6) traitements insecticides à intervalle de 14 jours à partir du $45^{\text {ème }}$ au $115^{\text {ème }}$ JAL (Ochou et al., 2012). Le deux premiers traitements se caractérisent par une restriction de l'utilisation des pyréthrinoïdes (Ochou et al., 2012).
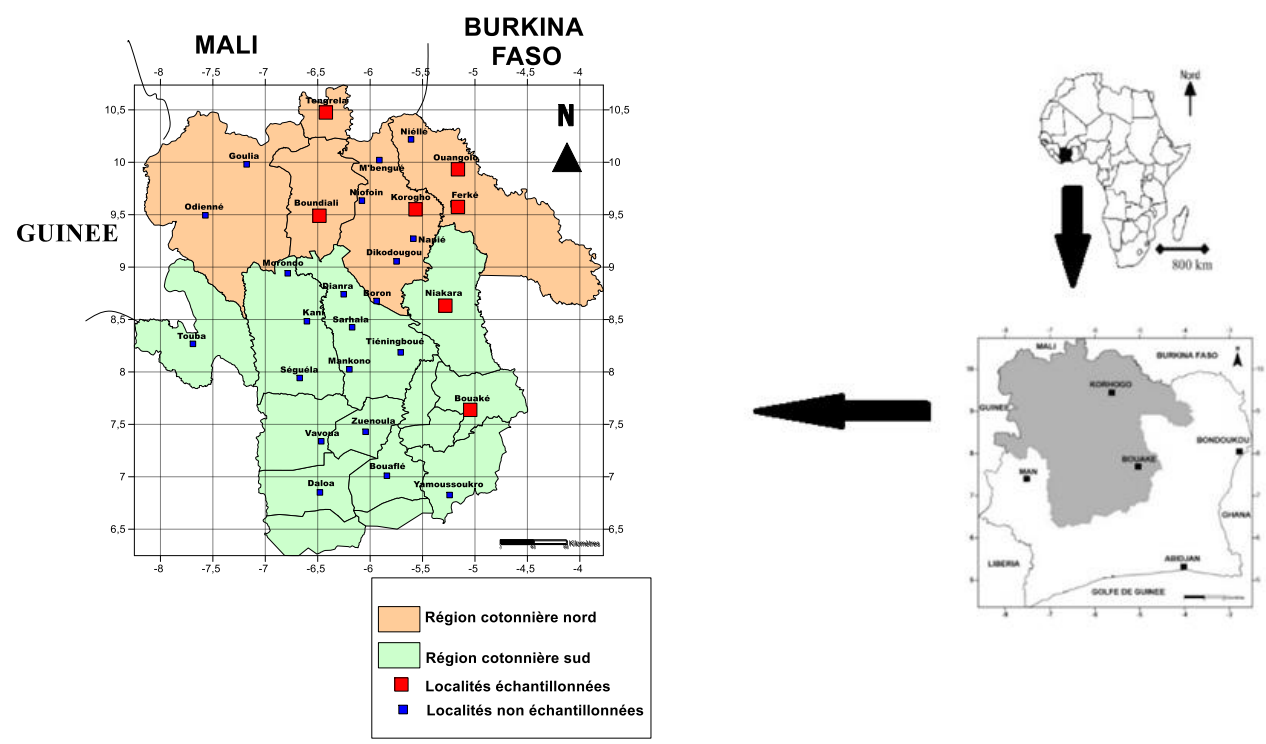

Figure 6: Localisation de la zone d'étude

Tableau 6: Insecticides utilisés pour la réalisation des tests toxicologiques

\begin{tabular}{cccc}
\hline $\begin{array}{c}\text { Familles de } \\
\text { molécules }\end{array}$ & matières actives & $\begin{array}{c}\text { Nom commercial du } \\
\text { produit }\end{array}$ & $\begin{array}{c}\text { Fabricants } \\
\text { ou distributeurs }\end{array}$ \\
\hline Néonicotinö̈de & Acétamipride $30 \mathrm{~g} . \mathrm{l}^{-1}$ & ASSAIL 30 SG & Dow Agrosciences \\
Organophosphoré & Profenofos 720 g. $\mathrm{l}^{-1}$ & CURACRON 720 EC & Syngenta \\
Pyréthrinö̈de & Lambda-cyhalothrine $100 \mathrm{~g} . \mathrm{l}^{-1}$ & KARATE ZEON 100 SC & Dow Agrosciences \\
Spinosyne & Spinosad 240 g. $\mathrm{l}^{-1}$ & GF-976 240 SC* & Dow Agrosciences \\
Sulfoximine & Sulfoxaflor 240 g. $\mathrm{l}^{-1}$ & GF-2032 240 SC* & Dow Agrosciences \\
\hline \multicolumn{4}{c}{}
\end{tabular}




\section{Méthode de dosage biologique par immersion des feuilles}

Les tests toxicologiques ont été réalisés selon la méthode utilisée par Houndété et $a l ., 2010$. Des rondelles de feuille de coton sont trempées pendant 10 secondes dans des solutions insecticides préparées à différentes concentrations avec trois répétitions par concentration, puis séchées à température ambiante. Les rondelles témoins sont trempées dans de l'eau distillée pour les formulations et dans de l'acétone diluée pour le grade technique. Une fois sèches, les rondelles de feuilles sont déposées dans les boites de pétri (de $5,5 \mathrm{~cm}$ de diamètre) contenant du gel d'agar $(7 \%)$, en prenant soin de disposer la face supérieure contre le gel. Vingt-cinq (25) à trente (30) insectes adultes de $B$. tabaci sont endormis au dioxyde de carbone et déposés dans les boites de pétri. L'on recouvre alors chaque boite avec son couvercle préalablement perforé de minuscules trous permettant l'aération. Lorsque les insectes se réveillent, la boite de pétri est renversée pour leur assurer une alimentation normale. Les préparations ainsi obtenues sont mises dans des conditions de $25 \pm 2{ }^{\circ} \mathrm{C}, 60 \%$ d'humidité relative et de photopériode naturelle (12h : 12h L/D). La mortalité est évaluée 48 heures après le traitement à l'aide d'une loupe à main. La mortalité des témoins est également enregistrée ; lorsque celle-ci est supérieure ou égale à $10 \%$, le test est annulé. Les résultats sont exprimés en pourcentage de mortalité corrigée à l'aide de la formule d'Abbott (Abbott, 1925).

\section{Analyse des données}

Le logiciel DL50 version 2.0 CIRAD-CA a servi pour les analyses des données à partir du modèle probit-log de Finney (1971). Un test Khi ${ }^{2}$ a été effectué à partir des valeurs des mortalités corrigées comprises entre 1 et $99 \%$. Le bon ajustement du modèle est indiqué par les valeurs non significatives des $\mathrm{Khi}^{2}\left(\chi^{2}\right)$ calculées au seuil de $5 \%$ (Olivier Gnankiné et al., 2002). Ceci a permis d'obtenir pour chaque matière active testée, la valeur en $\mathrm{mg} . \mathrm{l}^{-1} \mathrm{de}$ la concentration létale à $50 \%$ de la population testée $\left(\mathrm{CL}_{50}\right)$ avec une limite de confiance et une pente. Ainsi, la $\mathrm{CL}_{50}$ a permis d'évaluer le niveau de sensibilité à un insecticide pour les populations d'insecte de chaque localité. Plus sa valeurs est faible, plus la population testée est sensible à cet insecticide. Les $\mathrm{CL}_{50}$ calculées ont été comparées à l'aide d'analyses univariées de variance (ANOVA) grâce au logiciel IBM SPSS 22.0. Ceci a été fait après vérification de l'égalité des variances qui a indiqué homogénéité des données. Le test de Duncan a été effectué au seuil de $5 \%$ pour dégager les différents groupes ou sous-groupe homogènes. 


\section{Résultats}

Niveaux de sensibilité aux insecticides chez les populations de $B$ tabaci selon les localités

Les CL50 obtenues ont varié de 2,36 à 219,96 mg.1 ${ }^{-1}$, traduisant une très grande variabilité de la sensibilité aux insecticides des populations de $B$. tabaci selon le type de matière active testée et la localité. Les valeurs des pentes ont été relativement faibles variant de 0,5 à 1,79 (tableau 2).

\section{Niveaux de sensibilité à la lambdacyalothrine}

La sensibilité des mouches blanches à la lambda-cyhalothrine a été très variable d'une localité à une autre. Les valeurs des $\mathrm{CL}_{50}$ obtenues ont varié de 2, 65 à 136,71 mg. $\mathrm{l}^{-1}$. Les populations de Niakara (2,65 mg. $\left.\mathrm{l}^{-1}\right)$, Ferké $\left(\mathrm{CL}_{50}=4,18 \mathrm{mg} . \mathrm{l}^{-1}\right)$ et Ouangolo $\left(\mathrm{CL}_{50}=13,88 \mathrm{mg} \cdot \mathrm{l}^{-1}\right)$ ont été très sensibles à ce produit. En revanche les populations de Korhogo $\left(136,71 \mathrm{mg} . \mathrm{l}^{-1}\right)$ et Boundiali $\left(\mathrm{CL}_{50}=97,58 \mathrm{mg} . \mathrm{l}^{-1}\right)$ ont affiché une très faible sensibilité. Les populations de Bouaké $\left(\mathrm{CL}_{50}=41,05 \mathrm{mg} . \mathrm{l}^{-1}\right.$ ) ont montré une sensibilité moyenne. L'analyse de variance indique une différence significative $(\mathrm{F}=6,1 ; \mathrm{P}=0,02)$ entre les valeurs des CL50 obtenues (Tableau 2).

\section{Niveaux de sensibilité au profenofos}

On a observé une variabilité de la sensibilité des mouches blanches au profenofos dont les $\mathrm{CL}_{50}$ ont varié de 7,77 à 71,51 $\mathrm{mg} .1^{-1}$. Les populations de Korhogo (71,51 mg..$\left.^{-1}\right)$ ont affiché la plus faible sensibilité tandis que les populations de Ferké $\left(9,2 \mathrm{mg} . \mathrm{l}^{-1}\right)$ et Niakara $\left(7,77 \mathrm{mg} . \mathrm{l}^{-1}\right)$ ont relativement présenté une plus grande sensibilité. Les populations de Ouangolo (28,86 mg. $\left.\mathrm{l}^{-1}\right)$, Boundiali $\left(38,75 \mathrm{mg} \cdot \mathrm{l}^{-1}\right)$ et Bouaké $\left(49,63 \mathrm{mg} . \mathrm{l}^{-1}\right)$ ont présenté une sensibilité intermédiaire au profenofos (tableau 2). Il a été observé une différence significative entre les valeurs des $\mathrm{CL}_{50}$ obtenues $(\mathrm{F}=2,2 ; \mathrm{P}=0,04)$.

\section{Niveaux de sensibilité à l'acétamipride}

Les CL50 obtenues pour l'acétamipride ont varié de 7,31 à 219,96 $\mathrm{mgl}^{-}$ 1. Le test de Duncan montre une différence significative $(\mathrm{F}=3,75 ; \mathrm{P}=0,04)$ entre les valeurs obtenues. Ainsi, les populations de Ferké $\left(\mathrm{CL}_{50}=22,09 \mathrm{mg} . \mathrm{l}^{-}\right.$ $\left.{ }^{1}\right)$ et Niakara $\left(7,31 \mathrm{mg} .1^{-1}\right)$ ont été les plus sensibles tandis que les populations de Boundiali $\left(\mathrm{CL}_{50}=219,96 \mathrm{mg} . \mathrm{l}^{-1}\right)$ ont montré une très faible sensibilité à l'acétamipride. Les populations de Bouaké (35,85 mg. $\left.1^{-1}\right)$ Korhogo (44,46 mg. $\left.\mathrm{l}^{-1}\right)$ et Ouangolo $\left(105,05 \mathrm{mg} . \mathrm{l}^{-1}\right)$ ont été moyennement sensibles à ce produit (tableau 2).

\section{Niveaux de sensibilité au sulfoxaflor}

Les $\mathrm{CL}_{50}$ obtenues avec le sulfoxaflor ont été relativement faibles. Les populations de Boundiali $\left(\mathrm{CL}_{50}=4,51 \mathrm{mg} . \mathrm{l}^{-1}\right)$, Ferké $\left(\mathrm{CL}_{50}=3,35 \mathrm{mg} . \mathrm{l}^{-1}\right)$ et 
Bouaké $\left(\mathrm{CL}_{50}=3,53 \mathrm{mg} . \mathrm{l}^{-1}\right)$ ont montré une sensibilité élevée aux sulfoxaflor. Les populations de Korhogo $\left(\mathrm{CL}_{50}=11,94 \mathrm{mg} \mathrm{l}^{-1}\right.$ ) et de Ouangolo $\left(\mathrm{CL}_{50}=9,89\right.$ mg. $\left.1^{-1}\right)$ se distinguent avec une sensibilité relativement plus faible à ce produit (tableau 2). L'analyse de variance ne montre aucune différence significative $(\mathrm{F}=0,57 ; \mathrm{P}=0,71)$ entre les valeurs de $\mathrm{CL}_{50}$ obtenues.

\section{Niveaux de sensibilité au spinosad}

Les $\mathrm{CL}_{50}$ obtenues avec le spinosad ont été relativement faibles avec des valeurs variant de 1,3 et $3,71 \mathrm{mg} \cdot 1^{-1}$. On note tout de même que les populations de Boundiali se sont montrées plus sensibles au spinosad avec une $\mathrm{Cl}_{50}$ de $1,83 \mathrm{mg} . \mathrm{l}^{-1}$. Les populations de Bouaké $\left(\mathrm{CL}_{50}=3,71 \mathrm{mg} . \mathrm{l}^{-1}\right)$ ont été relativement moins sensibles au spinosad. Cependant, l'analyse statistique ne montre pas de différence significative $(\mathrm{F}=0,35 ; \mathrm{P}=0,86)$ entre les valeurs de $\mathrm{CL}_{50}$ Obtenues pour ce produit (tableau 2).

\section{Comparaison du niveau moyen de toxicité des insecticides testés}

Il a été observé une variation significative des $\mathrm{CL}_{50}$ moyennes calculées pour chaque insecticide dans toutes les localités $(\mathrm{F}=2,58 ; \mathrm{P}=0,04)$. Le sulfoxaflor et le spinosad se démarquent par leur très forte toxicité vis-àvis des mouches blanches avec des $\mathrm{CL}_{50}$ moyennes respectives de 5,88 et 2,97 mg. $1^{-1}$. Le Profenofos $\left(34,28 \mathrm{mg} \cdot \mathrm{l}^{-1}\right)$ et la lamdacyalothrine $\left(49,34 \mathrm{mg} \cdot \mathrm{l}^{-1}\right)$ ont montré une toxicité relativement faible tandis que l'acétamipride $\left(72,45 \mathrm{mg} .1^{-}\right.$ $\left.{ }^{1}\right)$ a présenté la toxicité la plus faible vis-à-vis des mouches blanches.

\section{Discussion}

Des pullulations spectaculaires de mouches blanches ont été observées ces dernières années surtout dans le Nord de la zone cotonnière de Côte d'ivoire (Didi et $a l ., 2018$ ). Il a donc été évalué au cours de cette étude, les niveaux de sensibilité des insectes de certaines localités à des insecticides couramment utilisés dans les programmes de protections phytosanitaire. Il s'agit de la lambdacyhalothrine (pyréthroïdes), le profenofos (organophosphoré) et l'acétamipride (néonicotinoïde). Une variabilité significativement importante de la sensibilité des mouches blanches à ces insecticides a été observée selon l'origine de la souche testée. Les populations d'insectes de Ferké et Niakara ont été les plus sensibles au profenofos, à l'acétamipride et à la lambdacyhalothrine. En revanche, les populations de Korhogo ont présenté la plus faible sensibilité au profenofos, et celle de Boundiali la plus faible sensibilité à l'acétamipride. 
Tableau 2. Niveau de sensibilité aux insecticides chez différentes populations de $B$ tabaci

\begin{tabular}{|c|c|c|c|c|c|c|}
\hline $\begin{array}{c}\text { Matières } \\
\text { actives }\end{array}$ & $\begin{array}{c}\text { Souches de } \\
\text { B. tabaci }\end{array}$ & $\overline{\mathbf{N}}$ & $\begin{array}{c}C_{50}\left(\mathrm{mg.l}^{-1}\right) \\
\text { L.C. } 95 \%\left(\text { CLLo }_{50}\right)\end{array}$ & $\overline{\chi^{2}}$ & $\overline{\text { Ddl }}$ & Pente \\
\hline \multirow{6}{*}{$\begin{array}{c}\text { Lambda } \\
\text { Cyhalothrin }\end{array}$} & Ouangolo & 256 & $13,88[6,9 ; 52,88] \mathrm{b}$ & 3,68 & 3 & 0,69 \\
\hline & Boundiali & 330 & $97,58[65,99 ; 375,84] \mathrm{a}$ & 2,21 & 3 & 0,58 \\
\hline & Korhogo & 322 & $136,71[37,7 ; 248,73] \mathrm{a}$ & 4,31 & 3 & 0,59 \\
\hline & Ferké & 251 & $4,18[1,76 ; 15,45] \mathrm{b}$ & 3,31 & 3 & 0,57 \\
\hline & Bouaké & 371 & $41,05[9,4 ; 181,67] \mathrm{ab}$ & 0,67 & 3 & 0,57 \\
\hline & Niakara & 351 & $2,65[0,20 ; 9,55] \mathrm{b}$ & 1,73 & 3 & 0,8 \\
\hline \multirow{6}{*}{ Profénofos } & Ouangolo & 369 & $28,86[7,8 ; 69,18] \mathrm{ab}$ & 6,62 & 4 & 0,9 \\
\hline & Boundiali & 351 & $38,75[10,29 ; 74,73] \mathrm{ab}$ & 3,32 & 3 & 1,38 \\
\hline & Korhogo & 380 & $71,51[9,27 ; 161,91] \mathrm{a}$ & 0,51 & 3 & 1,05 \\
\hline & Ferké & 250 & $9,2[1,28 ; 25,64] \mathrm{b}$ & 0,81 & 3 & 1,08 \\
\hline & Bouaké & 464 & $49,63[15,36 ; 92,23] \mathrm{ab}$ & 7,5 & 4 & 1,76 \\
\hline & Niakara & 352 & $7,77[3,91 ; 23,56] \mathrm{b}$ & 1,97 & 3 & 0,99 \\
\hline \multirow{6}{*}{ Acétamipride } & Ouangolo & 295 & $105,05[47,32 ; 205,46] \mathrm{ab}$ & 4,67 & 4 & 1,12 \\
\hline & Boundiali & 414 & $219,96[103,03 ; 316,54]$ a & 1,13 & 7 & 1,17 \\
\hline & Korhogo & 469 & $44,46[4,48 ; 145,78] \mathrm{ab}$ & 4 & 7 & 0,74 \\
\hline & Ferké & 270 & $22,09[3,1 ; 82,85] \mathrm{b}$ & 1,85 & 4 & 0,58 \\
\hline & Bouaké & 493 & $35,85[4,35 ; 171,31] \mathrm{ab}$ & 1,37 & 7 & 0,63 \\
\hline & Niakara & 398 & $7,31[2,58 ; 13,86] \mathrm{b}$ & 3,51 & 4 & 1,34 \\
\hline \multirow{6}{*}{ Sulfosaxflor } & Ouangolo & 293 & $9,89[3,94 ; 17,48]$ a & 4,41 & 4 & 1,52 \\
\hline & Boundiali & 245 & $4,51[0,82 ; 10,63]$ a & 2,97 & 3 & 1,13 \\
\hline & Korhogo & 266 & $11,94[4,58 ; 21,5] \mathrm{a}$ & 1,33 & 4 & 1,34 \\
\hline & Ferké & 206 & $3,35[0,26 ; 42,08] \mathrm{a}$ & 3,24 & 3 & 0,85 \\
\hline & Bouaké & 1365 & $3,53[0,22 ; 11,68]$ a & 1,90 & 3 & 0,92 \\
\hline & Niakara & 462 & $2,08[0,76 ; 9,41] \mathrm{a}$ & 3,89 & 4 & 0,73 \\
\hline \multirow{6}{*}{ Spinosad } & Ouangolo & 296 & $3,42[1,18 ; 5,85] \mathrm{a}$ & 5,25 & 3 & 1,79 \\
\hline & Boundiali & 235 & $1,83[0,31 ; 4,90] \mathrm{a}$ & 4,78 & 4 & 1,01 \\
\hline & Korhogo & 239 & $2,36[0,43 ; 5,84] \mathrm{a}$ & 0,76 & 4 & 1,12 \\
\hline & Ferké & 230 & $3,23[0,59 ; 7,53] \mathrm{a}$ & 4,42 & 4 & 1,22 \\
\hline & Bouaké & 1472 & $3,71[0,52 ; 10,39]$ a & 1,63 & 5 & 0,99 \\
\hline & Niakara & 463 & $3,28[0,53 ; 8,51] \mathrm{a}$ & 3,92 & 3 & 1,06 \\
\hline
\end{tabular}

$N=$ nombre d'insectes testés ; $95 \%$ L.C. : limites de confiance pour une probabilité fixée de $5 \% ; \mathrm{Cl}_{50}$ : Concentration létale tuant $50 \%$ de la population ; $\chi^{2}$ : Chi2 ; Ddl: degree de liberté.

Les valeurs de CLso suivies de même lettre ne sont pas significativement différentes entre.

Ces valeurs sont basées sur l'interaction des limites de confiance à $95 \%$. 


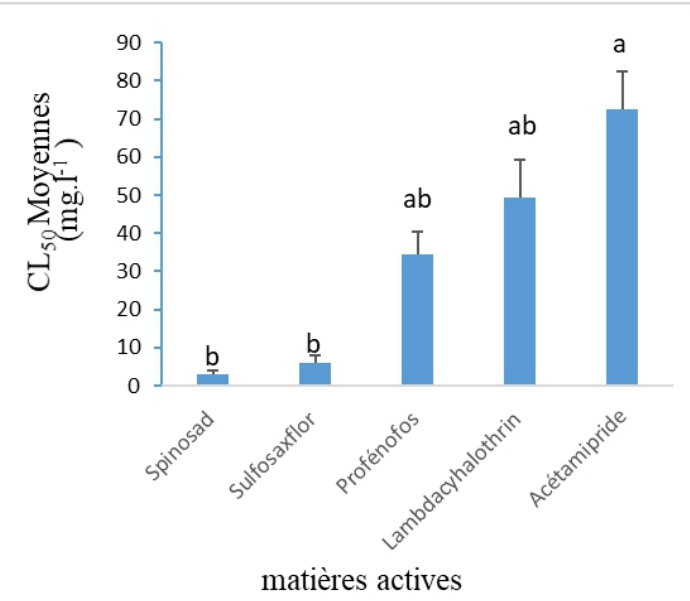

Figure 7: Comparaison de la toxicité moyenne des insecticides testés

Ces deux dernières populations ont montré toutes deux une très faible sensibilité à la lambdacyhalothrine. Kone et al. (2018) ont obtenues des résultats similaires, au cours d'une étude également effectuée sur des Cicadellidae en culture cotonnière de Côte d'Ivoire. Au cours de cette étude, il a été montré que les populations de Cicadelle de Korhogo et Boundiali ont présenté un taux de survie élevé suite à l'exposition à l'acétamipride, l'alphacyperméthrine et au profenofos, comparativement aux populations de Bouaké, Niakara, Ferké et Ouangolo. Selon ces auteurs, l'hétérogénéité génétique des populations testées est un facteur qui peut en partie expliquer ces différences dans les réponses aux insecticides testés. Les résultats obtenus indiquent des pentes relativement faibles dont les valeurs sont inférieures à 2 . Ahmad et al. (2002) ont également obtenu de faibles pentes au cours d'une étude toxicologique sur des populations de $B$. tabaci provenant du Pakistan. Ces auteurs attribuent ces faibles valeurs au comportement typique des souches de terrain qui présentent une hétérogénéité génétique qui influencerait les réponses des insectes aux insecticides. Les populations testées seraient alors génétiquement très hétérogènes. La faible sensibilité des populations de Korogho et Boundiali à l'acétamipride, la lambdacyalothrine et au profenofos signifie que ces populations sont beaucoup tolérantes à ces produits. Cela serait aussi lié aux pratiques culturales, plus précisément l'utilisation des insecticides. En effet, les Organophosphorés, les Pyréthrinoïdes et les néonicotinoïdes sont massivement utilisés depuis plus d'une vingtaine d'années en Afrique de l'Ouest, principalement contre les parasites agricoles et les vecteurs de maladie humaine. La pression de sélection exercée aurait provoqué un phénomène de résistance. Gnankiné et al., signalaient en 2002 la perte de sensibilité a l'acétamipride, la cyperméthrine et à une association de ces deux matières actives au Burkina Faso, pays frontalier à la Côte d'Ivoire. 
A cette période, $B$. tabaci était encore un ravageur secondaire sur le coton en Côte d'Ivoire (Hala \& Vassal, 1996). Toujours au Burkina Faso, Otoidobiga et al., (2003b) ont pu montrer une résistance de B. tabaci aux organophosphorés (méthamidophos et ométhoate) et à la cyperméthrine. Au cours d'une étude conjointement menée dans 3 pays d'Afrique de l'Ouest (Burkina Faso, Togo et Benin), Houndété et al., (2010) confirment cette forte résistance aux organophosphorés et aux pyréthrinoïdes des populations du Burkina Faso qui se sont avérées toutes aussi résistantes aux néonicotinoïdes tels que l'acétamipride et le thiametoxam. En l'absence de souche de référence dans le cas de notre étude, et le nombre de localités échantillonnés étant limité, il ne peut être véritablement évoqué un phénomène de résistance aux insecticides.

Néanmoins, la faible sensibilité des populations d'aleurodes de Boundiali, Korogho et Ouangolo à ces insecticides dits traditionnels observée semble être une transposition de la situation inhérente aux pays d'Afrique de l'ouest, dû à une utilisation massive et chronique de ces produits (Brévault et al., 2002; Ochou et al., 2012). Ce qui expliquerait en partie les fortes pullulations observées surtout à Korhogo et Ouangolo. Les niveaux de sensibilité établit pour l'acétamipride, le profenofos et lambdacyhalothrine sont ainsi révélateurs d'une perte de sensibilité à ces produits. Les valeurs des CL50 obtenues pourront servir de référence pour la surveillance parasitaire et des études ultérieures plus larges.

En outre, un phénomène très important serait aussi à l'origine de la perte de sensibilité aux insecticides chez les insectes en générale et chez $B$. tabaci en particulier dans les pays d'Afrique de l'Ouest. Il s'agit de l'utilisation anarchique de produits insecticides contrefaits ou non homologués qui inondent le marché des pesticides. Ce fait a été signalé sur le maraîcher par (Tarnagda et al., 2017) au Burkina Faso et par (Doumbia \& Kwadjo, 2009) en Côte d'Ivoire. Selon ces auteurs, la majorité des producteurs maraichers ne respectent pas le dosage recommandé des pesticides utilisés, contrairement aux cotonculteurs qui bénéficient d'un encadrement technique des sociétés cotonnières. Cependant, la plupart des insecticides destinés au coton sont détournés au profit des cultures maraichères (Fleischer et al., 1998 ; Tarnagda et $a l .$, 2017)). Les mouches blanches extrêmement polyphage, migrant entre les cultures, pourraient se trouver constamment en présence des mêmes matières actives et cela pourrait à la longue sélectionner des individus moins sensibles. Ce qui peut par exemple expliquer le faible niveau de sensibilité des populations de B. tabaci de Korhogo et Boundiali qui représentent dans le Nord, une grande zone de production maraichère comme le décrit Fromageot. (2008) et Oura. (2012). Ainsi, les mauvaises pratiques agricole en culture maraichère aurait une incidence en culture cotonnière et contribuerait à la 
pullulation de $B$. tabaci ces dernières années dans les agrosystèmes à base de coton signalé en Côte d'Ivoire par (Didi et $a l ., 2018$ ).

Par ailleurs, la comparaison du niveau moyen de toxicité des insecticides testés a montré une toxicité très faible relativement significative pour l'acétamipride, moyenne pour le profenofos et la lambdacyhalothrine et élevée pour le sulfoxaflor et le spinosad. Sur des populations israéliennes de B. tabaci, Horowitz et al. (2005) et Horowitz \& Ishaaya. (2014) ont montré une sélection du biotype MED-Q1 invasif par l'utilisation des néonicotinoïdes en conditions contrôlées et une résistance de plus en plus élevée pour ces populations à cette famille de molécule chimique. Le Biotype MED-Q1 s'est déjà signalé en sympatrie avec le Biotype MED-ASL dans les champs de coton aux Burkina (Houndété, 2010, Gnankiné et al.,2013 ; Gnankiné et al., 2018). La faible sensibilité à l'acétamipride observée dans la zone de production cotonnière en Côte d'Ivoire serait donc due à la sélection de Biotype MEDQ1 invasif. Mais cette hypothèse devra être vérifiée par des tests moléculaires sur les populations utilisées pour la réalisation des tests toxicologiques.

Le spinosad a l'avantage d'être de nature biologique. Cependant, il a un mode d'action voisin de celui des néonicotinoïdes. C'est un modulateur allostérique, tandis que les néonicotinoïdes sont des modulateurs compétitifs des récepteurs de l'acétylcholine (Sparks \& Nauen, 2015). Le risque de perte de sensibilité n'est également pas exclu concernant l'utilisation de ce produit. Le sulfoxaflor, bien que ayant également un mode similaire aux néonicotinoïdes, a présenté une toxicité relativement bonne vis-à-vis des populations de Ouangolo, Boundiali et Korhogo faiblement sensibles à l'acétamipride. Ces résultats sont proches de ceux de Sparks et al. (2013) et Wang et al. (2016) qui ont montré que le sulfoxaflor peut jouer un rôle important dans le control des individus de $\mathrm{B}$. tabaci déjà résistant aux néonicotinoïdes. Leur utilisation ainsi que celle des spinosynes doit être bien entendu raisonnée dans une stratégie intégrée. Le spinosad et le sulfoxaflor peuvent donc être des alternatives aux insecticides usuels et pourraient être bien utilisées dans la stratégie de gestion ou de prévention de résistance aux insecticides chez B. tabaci dans les agrosystèmes cotonniers en Côte d'Ivoire. .

\section{Conclusion}

Il ressort de cette étude, une très faible sensibilité des populations de B. tabaci à la lambdacyhalothrine, au profenofos et à l'acétamipride des populations de Boundiali, et Korhogo et Ouangolo dans le Nord. Au sud de la zone cotonnière, les populations de Niakara ont présenté des niveaux de sensibilité très élevée et celles de Bouaké, un niveau de sensibilités intermédiaires vis-à-vis de ces insecticides. Les niveaux de sensibilité obtenus pourront servir de niveau de référence pour la surveillance des infestations des mouches blanches. Le spinosad et le sulfoxaflor semblent être de meilleures 
options pour la gestion des populations de B. tabaci. Cette donnée nouvelle doit être prise en compte pour l'amélioration des programmes de protection en vue du contrôle ou de la gestion de la population de $B$. tabaci.

\section{References:}

1. Abbott, W. S. (1925). A Method of Computing the Effectiveness of an Insecticide. Journal of Economic Entomology, 18(2), 265-267. URL: https://doi.org/10.1093/jee/18.2.265a.

2. Ahmad, M., Arif, M. I., Ahmad, Z., \& Denholm, I. (2002). Cotton whitefly (Bemisia tabaci) resistance to organophosphate and pyrethroid insecticides in Pakistan. Pest Management Science, 58(2), 203-208. URL: https://doi.org/10.1002/ps.440.

3. Alon, M., Alon, F., Nauen, R., \& Morin, S. (2008). Organophosphates resistance in the B-biotype of Bemisia tabaci (Hemiptera: Aleyrodidae) is associated with a point mutation in an ace1-type acetylcholinesterase and overexpression of carboxylesterase. Insect Biochemistry and Molecular Biology, 38(10), 940-949. URL: https://doi.org/10.1016/j.ibmb.2008.07.007.

4. Brévault, T., Beyo, J., Nibouche, S., \& Vaissayre, M. (2002). La résistance des insectes aux insecticides: problématique et enjeux en Afrique centrale. In : Savanes africaines : des espaces en mutation, des acteurs face à de nouveaux défis. Actes du colloque, 27-31 mai 2002, Garoua, Cameroun, 7p.

5. Cahill, M., Byrne, F. J., Gorman, K., Denholm, I., \& Devonshire, A. L. (1995). Pyrethroid and organophosphate resistance in the tobacco whitefly Bemisia tabaci (Homoptera: Aleyrodidae). Bulletin of Entomological Research, 85(02), 181-187. URL: https://doi.org/10.1017/S0007485300034258

6. Didi, G. J. R., Kone, P. W. E., Ochou, G. E. C., Dekoula, S. C., Kouakou, M., Bini, K. K. N., Yao, M. D. S., Mamadou, D., \& Ochou, O. G. (2018). Évolution spatio-temporelle des infestations de la mouche blanche Bemisia tabaci (Gennadius, 1889) associées à la culture cotonnière en Côte d'Ivoire. Journal of Applied Biosciences, 121(1), 12202-URL:12210. https://doi.org/10.4314/jab.v121i1.10

7. Doumbia, M., \& Kwadjo, E. (2009). Pratiques d'utilisation et de gestion des pesticides par les maraîchers en Côte d'Ivoire : Cas de la ville d'Abidjan et deux de ses banlieues (Dabou et Anyama). Journal of Applied Biosciences, 18, 992-1002.

8. Eveleens, K. G. (1983). Cotton-insect control in the Sudan Gezira: analysis of a crisis. Crop Protection, 2(3), 273-287. URL: https://doi.org/10.1016/0261-2194(83)90002-9 
9. Farghly, S. F. (2013). Insecticide Sequences on Lambda-Cyhalothrin Resistance in Whitefly Bemisia tabaci (Genn.), 5.

10. Finney, D. J. (1971). Probit Analysis, third ed., 333 p. Cambridge, United Kingdom: Cambridge University Press, 333p.

11. Fleischer, G., Andoli, V., Coulibaly, M., \& Randolph, T. (1998). Analyse socio- économique de la filière des pesticides en Côte d'Ivoire. Rapport N6/F, Ministère de l'Agriculture et des Ressources Animales, Direction de la Protection des Végétaux et de la Qualité, Côte d'Ivoire, 112p.

12. Fromageot, A. (2008). Le maraîchage marchand dans le nord de la côte d'ivoire : une nouvelle forme d'agriculture urbaine loin de la ville? La diversité de l'agriculture urbaine dans le monde. Actes du colloque Les agricultures périurbaines, un enjeu pour la ville. ENSP, Université de Nanterre, 2008, 3, 95-108.

13. Gnankiné, O., Bassolé, I. H. N., Chandre, F., Glitho, I., Akogbeto, M., Dabiré, R. K., \& Martin, T. (2013). Insecticide resistance in Bemisia tabaci Gennadius (Homoptera: Aleyrodidae) and Anopheles gambiae Giles (Diptera: Culicidae) could compromise the sustainability of malaria vector control strategies in West Africa. Acta Tropica, 128(1), 7-17. URL: https://doi.org/10.1016/j.actatropica.2013.06.004

14. Gnankiné, Olivier, Hema, O., Namountougou, M., Mouton, L., \& Vavre, F. (2018). Impact of pest management practices on the frequency of insecticide resistance alleles in Bemisia tabaci (Hemiptera: Aleyrodidae) populations in three countries of West Africa. Crop Protection, 104, 86-91. URL: https://doi.org/10.1016/j.cropro.2017.10.020

15. Gnankiné, Olivier, Traoré, D., Dakouo, D., \& Sanon, A. (2002). Évolution de la sensibilité des adultes de Bemisia tabaci Gennadius (Homoptera: Aleyrodidae) vis-à-vis de quelques insecticides dans l'Ouest du Burkina Faso. Science et technique, Sciences naturelles et agronomie, 26(157-170), 14.

16. Gnankiné, Olivier, Traoré, D., Sanon, A., Traoré, N. S., \& Ouedraogo, A. P. (2007). Traitements insecticides et dynamique des populations de Bemisia tabaci Gennadius en culture cotonnière au Burkina Faso. Cahiers Agricultures, 16(2), 101-109. URL: https://doi.org/10.1684/agr.2007.0081

17. Hala, N., \& Vassal, J. M. (1996). Défense des cultures, Campagne 1995-1996, Rapport d'activité : opération 5132 «lutte intégrée contre les ravageurs du cotonnier ». Note technique, Centre National de Recherche Agronomique (CNRA), Côte d'Ivoire, non publié, 86p.

18. Horowitz, A. Rami, Kontsedalov, S., Khasdan, V., \& Ishaaya, I. (2005). Biotypes B and Q of Bemisia tabaci and their relevance to 
neonicotinoid and pyriproxyfen resistance. Archives of Insect Biochemistry and Physiology, 58(4), 216-225. URL: https://doi.org/10.1002/arch.20044

19. Horowitz, Abraham Rami, \& Ishaaya, I. (2014). Dynamics of biotypes B and Q of the whitefly Bemisia tabaci and its impact on insecticide resistance: Pest Management Science, 70(10), 1568-1572. URL: https://doi.org/10.1002/ps.3752

20. Horowitz, A.R., Denholm, I., \& Nichols, R. L. (2007). Managing Insecticide Resistance in Whiteflies and Aphid in cotton fields, $p$. 108-125.

21. Houndété, T. (2010). Résistance de Bemisia tabaci Gennadius (Homoptera: Aleyrodidae) aux insecticides: mécanismes enzymatiques et implications dans la gestion des populations. Thèse de Doctorat, Université de Lomé, 104p.

22. Houndété, T. A., Kétoh, G. K., Hema, O. S., Brévault, T., Glitho, I., \& Martin, T. (2010). Insecticide resistance in field populations of Bemisia tabaci (Hemiptera: Aleyrodidae) in West Africa. Pest Management Science, 66(11), 1181-1185. URL: https://doi.org/10.1002/ps.2008

23. Kady, H., \& Devine, G. J. (2003). Insecticide resistance in Egyptian populations of the cotton whitefly, Bemisia tabaci (Hemiptera: Aleyrodidae). Pest Management Science, 59(8), 865-871. URL: https://doi.org/10.1002/ps.687

24. Kone, P. W. E., Didi, R. J. G., Kouakou, M., Bini, K. K. N., Mamadou, D., \& Ochou, O. G. (2018). Susceptibility of cotton leafhopper Jacobiella facialis (Hemiptera: Cicadellidae) to principal chemical families: implications for cotton pest management in Côte d'Ivoire. Journal of Experimental Biology and Agricultural Sciences, 6(5), 774-781. URL: https://doi.org/10.18006/2018.6(5).774.781

25. Ochou, G. O., Doffou, M. N., N'Goran, E. K., \& Kouassi, K. (2012). Impact de la gestion de la résistance aux pyrethrinoïdes sur l'évolution spatio-temporelle des principaux lépidoptères carpophages du cotonnier en Côte d'Ivoire. Journal of Applied Biosciences, 53, 3831-3847.

26. Oliveira, M. R. V., Henneberry, T. J., \& Anderson, P. (2001). History, current status, and collaborative research projects for Bemisia tabaci. Crop Protection, 20(9), 709-723. URL: https://doi.org/10.1016/S02612194(01)00108-9

27. Otoidobiga, L. C., Vincent, C., \& Stewart, R. K. (2003a). Field Efficacy and Baseline Toxicities of Pyriproxifen, Acetamiprid, and Diafenthiuron Against Bemisia tabaci Gennadius (Homoptera: Aleyrodidae) in Burkina Faso (West Africa). Journal of Environmental 
Science and Health, Part B, 38(6), 757-769. URL: https://doi.org/10.1081/PFC-120025559.

28. Otoidobiga, L. C., Vincent, C., \& Stewart, R. K. (2003b). Susceptibility of field populations of adult Bemisia tabaci Gennadius (Homoptera: Aleyrodidae) and Eretmocerus sp (Hymenoptera: Aphelinidae) to cotton insecticides in Burkina Faso (West Africa). Pest Management Science, 59(1), 97-106. URL: https://doi.org/10.1002/ps.611

29. Prabhaker, N., Castle, S., Henneberry, T. J., \& Toscano, N. C. (2005). Assessment of cross-resistance potential to neonicotinoid insecticides in Bemisia tabaci (Hemiptera: Aleyrodidae). Bulletin of Entomological Research, 95(06). https://doi.org/10.1079/BER2005385

30. Quintela, E. D., Abreu, A. G., Lima, J. F. S., Mascarin, G. M., Santos, J. B., \& Brown, J. K. (2016). Reproduction of the whitefly Bemisia tabaci (Hemiptera: Aleyrodidae) B biotype in maize fields (Zea mays L.) in Brazil: First report of exotic B biotype whitefly adaptation to maize. Pest Management Science, 72(11), 2181-2187. URL: https://doi.org/10.1002/ps.4259

31. Sparks, T. C., \& Nauen, R. (2015). IRAC: Mode of action classification and insecticide resistance management. Pesticide Biochemistry and Physiology, 121, 122-128. URL: https://doi.org/10.1016/j.pestbp.2014.11.014

32. Sparks, T. C., Watson, G. B., Loso, M. R., Geng, C., Babcock, J. M., \& Thomas, J. D. (2013). Sulfoxaflor and the sulfoximine insecticides: Chemistry, mode of action and basis for efficacy on resistant insects. Pesticide Biochemistry and Physiology, 107(1), 1-7. URL: https://doi.org/10.1016/j.pestbp.2013.05.014

33. Tarnagda, B., Tankoano, A., Tapsoba, F., Sourabié, P. B., Abdoullahi, H. O., Djbrine, A. O., Drabo, K. M., Traoré, Y., \& Savadogo, A. (2017). Évaluation des pratiques agricoles des légumes feuilles : le cas des utilisations des pesticides et des intrants chimiques sur les sites marâichers de Ouagadougou, Burkina Faso. Journal of Applied Biosciences, 117(1), 11658-11668. URL: https://doi.org/10.4314/jab.v117i1.3

34. Tia, E. V., Lozano, P., Menut, C., Lozano, Y. F., Martin, T., Niamké, S., \& Adima, A. A. (2013). Potentialité des huiles essentielles dans la lutte biologique contre la mouche blanche Bemisia tabaci Genn. Phytothérapie, 11(1), 31-38. URL: https://doi.org/10.1007/s10298012-0736-8 
35. Vaissayre, M., Ochou, G. O., Hema, O. S. A., \& Togola, M. (2006). Quelles stratégies pour une gestion durable des ravageurs du cotonnier en Afrique subsaharienne ?, 15(1), 80-84.

36. Wang, R., Zheng, H., Qu, C., Wang, Z., Kong, Z., \& Luo, C. (2016). Lethal and sublethal effects of a novel cis-nitromethylene neonicotinoid insecticide, cycloxaprid, on Bemisia tabaci. Crop Protection, 83, 15-19. URL: https://doi.org/10.1016/j.cropro.2016.01.015 